\title{
Incidencia de hidronefrosis transitoria en reimplante vesicouretral abierto con técnica de Lich Gregoir en niños con reflujo vesicoureteral
}

\section{Incidence of Transient Hydroneprosis in Open Vesicouretral Reimplant with Lich Gregoir Technique in Children with Vesicoureteral Reflux}

\author{
Andrea Patrica Bolaños ${ }^{1}$ Carlos Alberto Larios ${ }^{1}$ Pablo Gonzalez Rodriguez ${ }^{2}$ Diana Carolina Franco ${ }^{1}$ \\ Jorge Andres Mora ${ }^{3}$ Yesica Yasmín Quiroz Madarriaga ${ }^{1}$

\footnotetext{
1 Departamento de Urología, Fundación Universitaria de Ciencias de la Salud, Hospital Infantil Universitario de San José de Bogotá, Bogotá, Colombia de San José de Bogotá, Bogotá, Colombia

3 Departamento de Urología, Unisanitas, Bogotá, Colombia
} \\ 2 Departamento de Urología Pediátrica, Hospital Infantil Universitario \\ Address for correspondence Yesica Quiroz Madarriaga, MD, \\ Hospital San José Calle $10 \# 18-75$, Bogotá, Colombia \\ (e-mail: yeyaquimapa@hotmail.com).
}

Urol Colomb 2019;28:255-259.

\section{Resumen}

\section{Palabras Clave}

- reflujo vesicoureteral

- reimplante ureterovesical extravesical

- Lich Gregoir

- hidronefrosis transitoria
Introducción y Objetivo La técnica de Lich Gregoir es actualmente la de elección para la corrección del reflujo vesicoureteral (RVU) de alto grado, con complicaciones como la obstrucción ureteral. La literatura reporta tasas del $6 \%$ al $28 \%$ de hidronefrosis transitoria, las cuales, en su gran mayoría, corresponden a un hallazgo temporal sin repercusión clínica. El objetivo de este trabajo es establecer la incidencia de hidronefrosis transitoria y la asociación con factores de riesgo.

Materiales y Metodos Realizamos una cohorte descriptiva retrospectiva, analizando 119 unidades ureterales reimplantadas mediante técnica de Lich Gregoir (LG) en menores de 18 años, entre Septiembre del 2009 y abril del 2017. Se excluyeron pacientes con megauréter primario, RVU secundario y cirugía previa para corrección de RVU. El análisis estadístico se realizó con Stata 13.0

Resultados Evaluamos 85 pacientes, 34 de ellos reimplantados de manera bilateral, para un total de 119 unidades ureterales. El promedio de edad fue de 4,4 años y el $68 \%$ fueron mujeres. El RVU grado IV fue el más prevalente con el $42 \%$. Las ecografías renales prequirúrgicas fueron normales en el $63 \%$ de los casos y el $14,2 \%$ presentó hidronefrosis transitoria con resolución a los 3 meses. No se reportaron casos de obstrucción ureteral. El grado de reflujo prequirúrgico no se asoció con hidronefrosis transitoria $(p=0,64)$ así como tampoco la lateralidad $(p=0,06)$.

Conclusión El reimplante vesicoureteral abierto con técnica de Lich Gregoir en nuestra institución, reporta tasas de hidronefrosis transitoria comparables a la literatura mundial. No se identificaron factores de asociación para hidronefrosis transitoria, diferentes a la técnica quirúrgica perse. received

February 8, 2018

accepted

April 24, 2018
DOI https://doi.org/

$10.1055 / \mathrm{s}-0038-1660527$

ISSN 0120-789X.

eISSN 2027-0119.
Copyright ( 2019 , Sociedad Colombiana License terms de Urología. Publicado por Thieme Revinter Publicações Ltda., Rio de Janeiro, Brazil. Todos los derechos reservados.

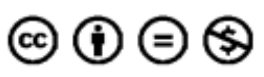




\begin{abstract}
Keywords

- vesicoureteral reflux

- vesicoureteral extravesical reimplant

- Lich Gregoir

- transient hydronephrosis

Introduction and Objective Lich Gregoir technique is currently the choice for correction of vesicoureteral reflux (VUR), with complications such as ureteral obstruction. Literature reports rates from $6 \%$ to $28 \%$ of transient hydronephrosis, from which, most correspond to a temporary finding without clinical repercussion. This study aims to establish the incidence of transient hydronephrosis and the association with risk factors.

Material and Methods In a descriptive retrospective cohort, we analyzed 119 ureteral units reimplanted with Lich Gregoir, under 18 years old, between September 2009 and April 2017. We have excluded patients with primary megaureter, VUR secondary and previous surgery for VUR correction. Statistical analysis was performed with Stata 13.0. Results We have evaluated 85 patients, 34 bilaterally reimplanted, totaling 119 ureteral units. Mean age was 4.4 years and $68 \%$ were women. VUR grade IV was the most prevalent with $42 \%$. Pre-surgical renal ultrasounds were normal in $63 \%$ of the cases, and $14.2 \%$ had transient hydronephrosis with resolution at 3 months. No case of ureteral obstruction was reported. Pre-surgical reflux grade was not associated with transient hydronephrosis $(p=0.64)$ as well as neither laterality $(p=0.06)$.

Conclusion Open Vesicoureteral reimplantaion with Lich Gregoir technique in our institution reports transient hydronephrosis rates similar to the literature. We have not found association factors with transient hydronephrosis, except for the surgical technique perse.
\end{abstract}

\section{Introducción}

El reimplante vesicoureteral con técnica de Lich Gregoir (LG) es el procedimiento quirúrgico de elección para la corrección abierta del RVU de alto grado con tasas de éxito que alcanzan el $98 \%$ y complicaciones poco frecuentes, dentro de las que prevalece la obstrucción ureteral con un $2 \%$ al $4 \%$ de los casos, la cual se presenta por edema, isquemia o rotación del uréter distal sobre su eje durante el reimplante. Retención urinaria del $8 \%$ al $15 \%$ por trauma del plexo pélvico durante la disección del uréter distal ${ }^{1}$ e hidronefrosis transitoria entre un $6 \%$ a un $28 \%$ de acuerdo a lo reportado en la literatura, ${ }^{2,3}$ sin representar lesiones a largo plazo en la función renal o patrón miccional de los pacientes.

En la literatura colombiana, no hay reportes sobre la incidencia de ese evento en nuestra población pediátrica, por lo que se pretende establecer la incidencia de la hidronefrosis transitoria de los pacientes llevados a reimplante vesicoureteral abierto con técnica de LG.

\section{Materiales y Métodos}

Se realizó una cohorte descriptiva retrospectiva incluyendo los pacientes menores de 18 años llevados a reimplante vesicouretral abierto con técnica de Lich Gregoir por RVU entre septiembre del 2009 y abril del 2017, encontrando 85 pacientes con 119 unidades ureterales (UU) evaluadas. Se excluyeron pacientes con megauréter primario, RVU secundario y cirugía previa para corrección de RVU.

Se evaluó en cada uno de ellos, género, edad al momento del procedimiento quirúrgico, grado de reflujo, lateralidad, función renal prequirúrgica, ecografía pre e hidronefrosis transitoria, ITU febril, disfunción miccional pre y POP, malformaciones urológicas asociadas, complicaciones, días de hospitalización, uso de sonda uretral y retención urinaria. Se definió el éxito quirúrgico de manera clínica por la ausencia de ITU POP y paraclínica con reporte de ecografía sin dilatación renal a los 3 meses del procedimiento quirúrgico.

Las ecografías se reportaron de acuerdo a la clasificación de hidronefrosis de la Sociedad Americana de Urología Fetal, y los grados de RVU de acuerdo a la clasificación del Comité Internacional del estudio del Reflujo mediante cistouretrografia miccional (CUMS). La CUMS no se solicita de manera rutinaria en los controles de nuestros pacientes y solo se realiza en caso de ITU febril, la cual se definió como la presencia de síntomas urinarios o respuesta inflamatoria sistémica con aislamiento positivo en Urocultivo.

Se definieron malos hábitos miccionales como: maniobras retenedoras, frecuencia urinaria menor de 8 veces por día y mala técnica miccional. El estreñimiento se definió como hábito intestinal mayor a 1 por día y deposiciones Bristol 1 , 2 y 3.

La función renal se informó en porcentaje diferencial de gammagrafías renales con DMSA y las complicaciones se reportaron de acuerdo a la clasificación de Clavien Dindo.

La base de datos se generó en formato Excel y el análisis estadístico descriptivo se reportó en rangos, promedios y frecuencias absolutas, utilizando STATA 13,0, con análisis univariado utilizando la prueba de Fisher para variables cualitativas y de Wilcoxon para las cuantitativas, con una $p<0,05$ como estadísticamente significativa. 


\section{Resultados}

Se encontraron 85 pacientes con RVU que se llevaron a corrección quirúrgica abierta por técnica extravesical en 119 unidades ureterales, con un promedio de edad 4,4 años, siendo la mayoría mujeres con un $68,2 \%$ de la población. El 98,2\% de los pacientes presentó ITU febril prequirúrgica, pero tan solo el $80 \%$ recibió profilaxis antibiótica, siendo la cefalexina el antibiótico más utilizado con un $68,2 \%$, seguido el TMP-SX con un 5,8\%. El resumen de las características de la población se da en la - Tabla $\mathbf{1}$.

El RVU grado IV fue el más frecuente en un $42 \%$ de las unidades ureterales y 30,25\% grado III, siendo la mayoría bilaterales en un $40 \%$, con una función renal promedio por DMSA de $45,1 \%$ (15\% a 100\%). En cuanto a las ecografías renales prequirúrgicas, el $63 \%$ de las UU no presentaba hidronefrosis (HNF) y el 16,8\% tenía HNF grado I ( - Tabla 2).

Respecto al control POP de las ecografías, el 48,7\% permaneció igual, el 28,6\% mejoró y el 22,7\% reportó progresión de la hidronefrosis, del cual el 14,2\% se trató de una hidronefrosis transitoria que mejoró en el transcurso de los siguientes 3 meses POP, el 7,5\% no tuvo controles posteriores y en el $0,8 \%$ la progresión fue definitiva, paciente en el que se documentó recidiva del RVU (-Fig. 1).

Al buscar asociaciones entre la hidronefrosis transitoria y características como: grado de RVU prequirúrgico $(p=0,64)$, lateralidad $(p=0,06)$, edad como $>2$ años $0<2$ años $(p=1,00)$

Tabla 1 Descripción de la población

\begin{tabular}{|c|c|c|}
\hline & $\mathrm{n}=85$ & $\%$ \\
\hline \multicolumn{3}{|l|}{ Género } \\
\hline Femenino & 58 & 68,2 \\
\hline Masculino & 27 & 31,7 \\
\hline Edad (promedio) & 4,4 años & \\
\hline ITU febril prequirúrgico & 84 & 98,8 \\
\hline Profilaxis antibiótico & 68 & 80 \\
\hline \multicolumn{3}{|l|}{ Lateralidad } \\
\hline Derecho & 25 & 29,4 \\
\hline Izquierdo & 26 & 30,6 \\
\hline Bilateral & 34 & 40 \\
\hline \multicolumn{3}{|l|}{ Disfunción miccional } \\
\hline Prequirúrgica & 39 & 45,8 \\
\hline Posoperatoria & 15 & 17,6 \\
\hline \multicolumn{3}{|l|}{ Malformaciones urológicas } \\
\hline Ninguna & 66 & 77,6 \\
\hline Divertículos vesicales & 10 & 11,8 \\
\hline Doble sistema colector & 7 & 8,2 \\
\hline Ureterocele & 1 & 1,2 \\
\hline Riñón ectópico & 1 & 1,2 \\
\hline Tiempo quirúrgico (promedio) & 85 & \\
\hline Estancia hospitalaria (promedio) & 1,6 días & \\
\hline
\end{tabular}

Tabla 2 Descripción prequirúrgica unidades ureterales

\begin{tabular}{|c|c|c|}
\hline & $n=119$ & $\%$ \\
\hline \multicolumn{3}{|l|}{ Grado de reflujo } \\
\hline Grado I & 3 & 2,5 \\
\hline Grado II & 13 & 10,9 \\
\hline Grado III & 36 & 30,2 \\
\hline Grado IV & 50 & 42 \\
\hline Grado V & 17 & 14,4 \\
\hline $\begin{array}{l}\text { Función renal DMSA del riñón } \\
\text { afectado(promedio) }\end{array}$ & 45,1 & \\
\hline \multicolumn{3}{|l|}{ Ecografía renal prequirúrgica } \\
\hline Normal & 75 & 63 \\
\hline HNF grado I & 20 & 16,8 \\
\hline HNF grado II & 15 & 12,6 \\
\hline HNF grado III & 6 & 5,1 \\
\hline HNF grado IV & 3 & 2,5 \\
\hline
\end{tabular}

y disfunción miccional prequirúrgica de esos pacientes, no se encontró una relación estadísticamente significativa.

En el seguimiento 7 pacientes presentaron ITU, de los cuales 6 continuaban con disfunción miccional y entre ellos se presentó el único paciente con recidiva que requirió reintervención con técnica intravesical, y el paciente restante cursa con sospecha de vejiga neurogénica por disrrafismo oculto.

Respecto a los días de hospitalización, fueron en promedio 1,6 días y el 88,2\% de los pacientes requirió sonda uretral en el pop con 3 días en promedio de uso y una incidencia acumulada de retención urinaria del 10,5\%.

En cuanto a las complicaciones, solo se presentaron en 5 pacientes (5,6\%), 2 de ellos Clavien Dindo grado I y 3 grado II al tratarse de un sangrado que requirió transfusión, otro paso de sonda uretral por hematuria y otro una infección de sitio operatorio que no requirió intervención.

\section{Discusión}

La técnica extravesical de Lich-Gregoir descrita inicialmente en la década de los 60, seguida de una modificación por Lapointe en 1998 para facilitar la realización del túnel submucoso, es el Gold estándar para el manejo de RVU de alto grado con tasas de éxito que alcanzan hasta el $98 \%{ }^{4}$ Las complicaciones reportadas son bajas, siendo una de las más importantes la obstrucción ureteral, que puede alcanzar una frecuencia del $4 \%$, dentro de las cuales los casos de hidronefrosis transitoria, oscilan entre el 6 hasta el 28\%. En nuestro estudio se alcanzó una tasa de hidronefrosis transitoria de $14,2 \%$, lo cual se encuentra dentro de los rangos reportados en la literatura, presentándose generalmente por edema del detrusor en la unión ureterovesical. ${ }^{5}$

En cuanto a las asociaciones descritas para presentar hidronefrosis transitoria, Lee y col., describieron que los pacientes menores de 2 años tenían mayor riesgo de presentar ese evento $(p=0,003)$, mientras que el grado del 


\begin{tabular}{|c|c|c|c|c|c|c|}
\hline \multicolumn{2}{|c|}{} & \multicolumn{5}{|c|}{ HNF POST } \\
\cline { 2 - 7 } \multicolumn{2}{|c|}{} & $\mathbf{0}$ & $\mathbf{1}$ & $\mathbf{2}$ & $\mathbf{3}$ & $\mathbf{4}$ \\
\hline \multirow{4}{*}{} & $\mathbf{0}$ & 55 & 13 & 3 & 3 & 1 \\
\cline { 2 - 7 } & $\mathbf{1}$ & 11 & 2 & 6 & 0 & 1 \\
\cline { 2 - 7 } & $\mathbf{2}$ & 9 & 5 & 1 & 0 & 0 \\
\cline { 2 - 7 } $\begin{array}{c}\text { HNF } \\
\text { PRE }\end{array}$ & $\mathbf{3}$ & 0 & 4 & 2 & 0 & 0 \\
\cline { 2 - 7 } & $\mathbf{4}$ & 0 & 1 & 2 & 0 & 0 \\
\hline
\end{tabular}

\begin{tabular}{|l|l|l|}
\hline Progresó & 27 & $22,7 \%$ \\
\hline Igual & 58 & $48,7 \%$ \\
\hline Resolvió & 34 & $28,6 \%$ \\
\hline
\end{tabular}

Fig. 1 Evolución de la ecografía Prequirúrgica al mes POP.

RVU, la lateralidad, la función renal por DMSA y la presencia de divertículos vesicales, no presentaba ninguna relación. ${ }^{3}$ En este estudio, no se encontró una asociación con el grupo etario $(p=1,00)$, así como tampoco con el grado de RVU prequirúrgico $(p=0,64)$, la lateralidad $(p=0,06)$ y la disfunción miccional prequirúrgica de los pacientes, siendo la técnica quirúrgica perse el único evento desencadenante.

Brian y cols., en 2012 publicaron un estudio de 936 pacientes llevados a reimplante abierto (utilizaron tanto técnica intravesical como extravesical), y encontraron que los pacientes con HNF previa (OR 8,5, IC 95\% 6,01-12,1) y los RVU de alto grado (OR 3,38, IC 95\% 2,28-5,01) eran factores que incrementaban la aparición de HNF POP, sin especificar si se trataba o no de un evento transitorio. ${ }^{2}$ En el análisis univariado la HNF prequirúrgica fue estadísticamente significativa como predictor de persistencia de la misma en el POP $(p=0,003)$. En este estudio reportaron hidronefrosis POP en el $21 \%$ de los pacientes, incluyendo aquellos que persistieron con hidronefrosis desde el prequirúrgico y los pacientes que desarrollaron de novo en el POP, informando mejoría de la misma al año de seguimiento en el 70,6\%. Además, los pacientes con HNF de novo se resolvieron espontáneamente más frecuentemente que aquellos que ya cursaban con HNF previa. ${ }^{2}$ En nuestra serie, el 22,4\% de los pacientes presentó progresión de la HNF inicial o aparición postquirurgica de novo. La resolución de la HNF se evidenció en un 28,6\% de los pacientes en su mayoría a los 3 meses, siendo más rápida la resolución de aquellos que no cursaban con HNF prequirúrgica.

Barrieras y cols., en 2000 publicaron un trabajo sobre 723 unidades ureterales que se llevaron a reimplante extravesical, reportando una tasa de hidronefrosis transitoria del 7,2\% a las 6 semanas y 0,005\% al año; sugirió además, que ante un paciente con hipertensión arterial, dolor abdominal, elevación de azoados, disminución de la producción de orina o episodios repetitivos de emesis, se ameritaban estudios adicionales para descartar un proceso obstructivo adyacente. ${ }^{6}$ La tasa reportada de obstrucción ureteral en general para esa técnica quirúrgica, oscila entre el $2 \%$ y el $4 \%{ }^{1}$ En nuestra revisión, no se reportaron casos de obstrucción ureteral y el tiempo de resolución de la HNF transitoria fue mayor, debido al seguimiento menos frecuente de nuestra población, por la baja adherencia de los padres y tiempos de espera para las citas ambulatorias y autorizaciones.
En el estudio de Blais et col., en 2017, mencionan que la retención urinaria transitoria descrita en la literatura para el abordaje extravesical, es secundaria a la denervación durante la detrusotomia, que puede alterar la contractilidad del detrusor y con ello causar tanto retención urinaria como disfunción miccional. ${ }^{7}$

En nuestra serie, la tasa de retención fue de un 10,5\%, siendo la frecuencia más alta encontrada en la literatura la de $15 \%{ }^{1}$

Respecto a las malformaciones urológicas en nuestra población, tan solo el $22,4 \%$ presentó alguna anomalía asociada, siendo la más frecuente los divertículos vesicales, seguida de los dobles sistemas colectores, encontrándose solo un paciente con ITU POP con alteración anatómica, que además cursaba con disfunción miccional. Jayanthi y col., en 1995, no encontraron ningún efecto de la corrección del divertículo sobre la aparición de la obstrucción ureteral $\mathrm{POP}^{8}$

El tiempo quirúrgico reportado en la literatura es de 52 minutos en promedio para reimplantes unilaterales. En nuestra revisión, la media de duración fue de 85 minutos (56-97 min), ${ }^{1}$ teniendo en cuenta que la mayoría de la población se intervino por RVU bilateral. La estancia hospitalaria promedio de la literatura está reportada en 6,5 días con rangos que oscilan entre los 3 y 9 días, ${ }^{1}$ siendo mucho más bajo el promedio de nuestra población con 1,6 días.

Las limitaciones de este estudio principalmente se encuentran en su carácter retrospectivo, ya que introduce sesgos de selección e información y el tiempo promedio de seguimiento fue de 27 meses, lo cual no permite determinar desenlaces a largo plazo.

\section{Conclusión}

El reimplante vesicoureteral abierto con la técnica de Lich Gregoir en nuestra institución, reporta tasas de hidronefrosis transitoria comparables a la literatura mundial. Se pudo observar una asociación estadísticamente significativa entre la hidronefrosis prequirúrgica y la aparición de hidronefrosis transitoria postoperatoria en los pacientes que fueron intervenidos.

\section{Responsabilidades Éticas}

Protección de personas y animales. Los autores declaran que para esta investigación, no se han realizado experimentos en seres humanos ni en animales. 
Confidencialidad de los datos. Los autores declaran que han seguido los protocolos de su centro de trabajo sobre la publicación de datos de pacientes.

Derecho a la privacidad y consentimiento informado. Los autores declaran que en este artículo no aparecen datos de pacientes.

\section{Referencias}

1 Heidenreich A, Ozgur E, Becker T, Haupt G. Surgical management of vesicoureteral reflux in pediatric patients. World J Urol 2004;22 (02):96-106

2 Rosman BM, Passerotti CC, Kohn D, Recabal P, Retik AB, Nguyen HT. Hydronephrosis following ureteral reimplantation: when is it concerning? J Pediatr Urol 2012;8(05):481-487
3 Lee YS, Im YJ, Jung HJ, Hah YS, Hong CH, Han SW. Does hydronephrosis after extravesical ureteral reimplantation deteriorate renal function? J Urol 2012;187(02):670-675

4 Lapointe SP, Barrieras D, Leblanc B, Williot P. Modified LichGregoir ureteral reimplantation: experience of a Canadian center. J Urol 1998;159(05):1662-1664

5 Aboutaleb H, Bolduc S, Bägli DJ, Khoury AE. Correlation of vesicoureteral reflux with degree of hydronephrosis and the impact of antireflux surgery. J Urol 2003;170(4 Pt 2):1560-1562, discussion 1562

6 Barrieras D, Lapointe S, Reddy PP, et al. Are postoperative studies justified after extravescial ureteral reimplantation? J Urol 2000; 164(3 Pt 2):1064-1066

7 Blais AS, Bolduc S, Moore K. Vesicoureteral reflux: From prophylaxis to surgery. Can Urol Assoc J 2017;11(1-2Suppl1):S13-S18

8 Jayanthi VR, McLorie GA, Khoury AE, Churchill BM. Extravesical detrusorrhaphy for refluxing ureters associated with paraureteral diverticula. Urology 1995;45(04):664-666 